\title{
Procedimiento metodológico para la implementación de soluciones FinTech en PyMEs de comercio
}

\section{Methodological procedure for implementation of FinTech solutions in commerce SMEs}

\author{
Patricio Medina-Chicaiza \\ patricio_medina@hotmail.com \\ Universidad Técnica de Ambato \\ Ecuador \\ http://orcid.org/0000-0002-2736-8214 \\ Lisseth Carolina León-Aguagallo \\ lleon9320@uta.edu.ec \\ Universidad Técnica de Ambato \\ Ecuador \\ http://orcid.org/0000-0002-7266-2615
}

Recibido: 1 de junio del 2019

Aprobado: 21 de junio del 2019

\section{RESUMEN}

El objetivo del trabajo es proponer con un procedimiento metodológico para la implementación de un canal de pago digitalizado relacionado a las PyMEs. Empresas en desarrollo que deseen adoptar esta alternativa para distinguirse en un mercado cada vez más competitivo. El marco conceptual se sustenta a partir de recopilación, revisión, y análisis de referencias en español e inglés de libros, artículos indexados y estudios de organismos nacionales e internacionales referentes al tema. Así mismo, la metodología empleada se fundamenta con la aplicación de observación directa y entrevistas a empresas proveedoras FinTech de sistemas de tipo pago (FTP). Como resultado, se destaca el análisis comparativo entre los proveedores implicados; y se propone las siguientes fases: Cumplimiento de requisitos legales, Selección, Registro, Validación, Integración, Capacitación y pruebas, Difusión, Seguimiento y Evaluación. 
Descriptores: tecnología de la información; recursos financieros; finanzas y comercio; empresa

\section{ABSTRACT}

The objective of this paper is to propose a methodological procedure for implementation of a digitized payment channel related to SMEs. Developing companies that wish to adopt this alternative to distinguish themselves in an increasingly competitive market. The conceptual framework is based on the compilation, review, and analysis of references in Spanish and English of books, indexed articles, and studies by national and international organizations on the subject. Likewise, the methodology used is based on direct observation and interviews with Payment processing \& Fintech Solutions providers (FTS). Finally, the results highlights the comparative analysis between the suppliers involved; and the following phases are proposed: Compliance with legal requirements, Selection, Registration, Validation, Integration, Training and testing, Diffusion, Monitoring and Evaluation.

Descriptors: information technology; financial resources; finance and trade; enterprises

\section{INTRODUCCIÓN}

A nivel mundial, día a día son más las empresas que suman a sus atractivos, servicios apoyados en las tecnologías emergentes como las FinTech (Tecnologías financieras), siendo sus beneficiarios principales las Pequeñas y Medianas Empresas (PyMEs). Para Cleri (2007), las PyMEs, son empresas generalmente jóvenes de propiedad familiar, usualmente gestionadas por su creador/fundador, que en ocasiones tienden a mantenerse inestables debido a la exclusión financiera, principalmente a causa de su corto historial crediticio o el riesgo elevado que conlleva para las entidades bancarias tradicionales. Tundidor (2017) añade, que son máquinas de potencia y versatilidad, con remarcadas características de facilidad y agilidad para: a) tomar decisiones, b) hacer cambios a sus procesos, herramientas de gestión o información, c) generar compromiso en sus colaboradores, d) implementar herramientas tecnológicas, y e) establecer relaciones cercanas con sus clientes; lo que las convierte en clientes potenciales de soluciones tecnológicas como las FinTech. 
Al existir sinnúmero de PyMEs, debido a su diversidad varios autores (Ferraro et al., 2011) han definido que su tipificación se basa principalmente en parámetros de: facturación anual, número de personas empleadas, o una combinación de estos. En el caso de Ecuador, el Instituto Nacional de Estadísticas y Censos (INEC, 2017), entidad reguladora de las estadísticas oficiales del país para la toma de decisiones de política pública, ha determinado la siguiente clasificación:

Tabla 1. Categorización de empresas en Ecuador

\begin{tabular}{|c|c|c|c|c|c|}
\hline & Micro empresa & Pequeña & Mediana "A" & Mediana "B" & Grande \\
\hline Ventas & $\begin{array}{l}\text { Menor o igual a } \\
\quad \$ 100.000\end{array}$ & $\begin{array}{c}\mathrm{De} \\
\$ 100.001 \mathrm{a} \\
\$ 1^{\prime} 000.000\end{array}$ & $\begin{array}{c}\mathrm{De} \\
\$ 1^{\prime} 000.001 \mathrm{a} \\
\$ 2^{\prime} 000.000\end{array}$ & $\begin{array}{c}\mathrm{De} \\
\$ 2^{\prime} 000.001 \mathrm{a} \\
\$ 5^{\prime} 000.000\end{array}$ & $\begin{array}{c}\mathrm{De} \\
\$ 5^{\prime} 000.00 \\
1 \text { en } \\
\text { adelante }\end{array}$ \\
\hline Personal & De 1 a 9 & De 10 a 49 & De 50 a 99 & De 100 a 199 & $\begin{array}{c}\text { De } 200 \text { en } \\
\text { adelante }\end{array}$ \\
\hline
\end{tabular}

Fuente: (INEC, 2017)

La tabla anterior refleja que, las PyMEs poseen de 10 a 199 trabajadores, y su ingreso por ventas anuales se sitúa en una escala de 100.001 a 5’000.000 de dólares americanos, no obstante se debe considerar criterios que fluctúan según la realidad de cada país y su nivel de desarrollo.

En referencia a estudios del Banco Mundial (2018) se afirma que las PyMEs en Iberoamérica representan casi el $90 \%$ del total de empresas existentes, entre un 50 y $75 \%$ de los empleos, y al menos un $50 \%$ del Producto Interno Bruto (PIB) de un país, por tanto, para competir en un mercado saturado de PyMEs se encuentra necesaria la innovación empresarial.

Al respecto, Villaseca (2016), manifiesta que la innovación empresarial plantea soluciones relevantes para los clientes y denota la preocupación de las empresas por comprender y adaptarse a los cambios del entorno, entender a su consumidor actual; Tundidor (2017), añade que busca transformar en valor agregado una oportunidad de 
Revista Arbitrada Interdisciplinaria KOINONIA

Año IV. Vol IV. N ${ }^{\circ}$. Julio - Diciembre 2019

Hecho el depósito de Ley: FA2016000010

ISSN: 2542-3088

FUNDACIÓN KOINONIA (F.K). Santa Ana de Coro. Venezuela.

Patricio Medina-Chicaiza; Lisseth Carolina León-Aguagallo

mejora a través de cambios, e involucra poner en marcha ideas mediante un camino planificado, de múltiples formas como en: diseño, distribución, procesamiento de información, comunicación, acceso al mercado, experiencia del usuario, entre otras. Por su parte, Maya (2019) indica que va de la mano con los cambios y la evolución del contexto en el que se desarrolla el sector empresarial, situándose la capacidad de cambio como una de las características más necesarias para la mejora empresarial. A pesar de las creencias utópicas que han sugerido que la innovación está predestinada solo a grandes empresas, la innovación en PyMEs se convierte en un factor estratégico para brindar un mejor producto o servicio, así lo señalan Martínez y El Kadi (2019), por tanto, se vuelve indispensable para sobresalir en un mercado altamente competitivo.

La innovación en términos FinTech (tecnología-financiera), McWaters \& Bruno (2017) manifiestan que en los últimos años se han dado lugar empresas intermediarias especializadas en tecnología financiera principalmente para el segmento PyMEs; de acuerdo con Orueta et al. (2017) éstas cuentan con capacidad técnica para desarrollar nuevos procesos, aplicaciones, modelos de negocio y productos relacionados con los servicios financieros, que asociados con la banca generan: a) Reducción de costos, b) Diferenciación y retención de clientes, c) Transparencia, d) Mejora de la experiencia del usuario e) Capacidad de innovación en productos y servicios financieros; autores como Maldonado y Naranjo (2017) señalan que en general, las FinTech giran en torno a cuatro áreas principales: Gestión de finanzas, Pagos y transacciones, Nuevos planteamientos de financiamiento y otorgamiento de créditos; $y$, Plataformas de consultoría y comercialización para inversiones. Estos aportes permiten vislumbrar a las soluciones FinTech como una herramienta que potenciará una ventaja competitiva en las empresas que las apliquen.

El rol de esta disyuntiva en Ecuador, se presenta como un desafío en el ámbito empresarial, ya que de acuerdo con estudios del Inter-American Development Bank (IDB) \& Finnovista (2017), el ecosistema FinTech ecuatoriano destaca la participación 
de tres modelos de negocios: gestión financiera empresarial (32\%), tecnologías empresariales para instituciones financieras $(16 \%)$, y, pagos y remesas $(16 \%)$, sobre otros que representan el porcentaje restante, además, a diferencia de otros países latinoamericanos en desarrollo, el país cuenta con apenas 31 empresas dedicadas a brindar servicios financiero-tecnológicos, por lo que se presume que hasta el momento solo un bajo porcentaje de empresas nacionales han adaptado soluciones Fintech.

Resulta oportuno mencionar que las soluciones innovadoras que proveen las FinTech resultarían de apoyo a la consecución de objetivos, mejora de procesos, y experiencia del usuario con un servicio de valor agregado a través de múltiples alternativas, como la de canales de pago dada a través de las plataformas virtuales, siendo las PyMEs de comercio de Ecuador un mercado potencial para la inclusión de esta alternativa; en tal razón, el estudio de soluciones FinTech favorece la innovación a través de la comprensión de dichas soluciones.

En Ecuador, la situación problemática que se identifica por observación directa es que, pocas PyMEs de comercio cuentan con una solución FinTech de tipo pago (FTP) implementada, lo que involucra el desconocimiento de herramientas de éste tipo; a su vez, se evidencia que no cuentan con un departamento especializado en desarrollo de plataformas virtuales de pago propias, sino que priorizan su personal en áreas de venta, contabilidad u otros, lo que conlleva a una barrera en el uso de nuevas soluciones tecnológicas que apoyen a la empresa. Por tanto, el análisis de las plataformas FTP, resulta una cuestión de interés que hasta la fecha no había sido abordada en estudios en el entorno ecuatoriano; con esto, se pretende contribuir a su entendimiento y brindar un marco de referencia para las empresas que deseen adoptar esta nueva apuesta tecnológica en el país. De este modo los beneficiarios directos del presente estudio son las PyMEs de comercio, entre tanto los indirectos son los profesionales relacionados con las áreas de gestión de negocios, marketing, organización de empresas y afines, y por supuesto los clientes que accederán a un nuevo canal de pago virtual. 
Con estos antecedentes, el presente documento desarrolla un análisis comparativo entre las plataformas FTP existentes en Ecuador, y aporta un procedimiento metodológico para su implementación en PyMEs de comercio.

\section{METODOLOGÍA}

Para la presente investigación, el marco conceptual se sustenta a partir de la recopilación, revisión, y análisis de referencias en español e inglés de libros, artículos indexados en bases de datos (SciELO, Springer Journals, Science Direct, Dialnet, Google Académico), y estudios de organismos nacionales e internacionales: Instituto Nacional de Estadísticas y Censos (INEC), EKOS Ecuador, Cámara de Comercio de Bogotá, Asociación Española para la Calidad, Asociación de Supervisores Bancarios de las Américas (ASBA), Banco Mundial, BBVA Innovation Center, Olyver Wyman \& Corporación Interamericana de Inversiones - CII, Inter-American Development Bank (IDB) \& Finnovista; el estudio asume elementos estratégicos de las FinTech como: características principales, modelos y agentes del ecosistema, para posteriormente obtener conclusiones a través del método de análisis-síntesis.

Para la comparación de las plataformas FTP vigentes en Ecuador, en principio se revisaron los datos del estudio "Radar FinTech Ecuador" realizado por Finnovista (2017), donde se identificaron tres empresas de tipo Sistemas de pago; posteriormente se procedió con observación directa en sus sitios web, para contrastar la información en base a 11 criterios establecidos por las propias empresas. Se detalla en la siguiente tabla:

Tabla 2. Criterios revisados los sitios web de FinTech de pago (FTP).

\begin{tabular}{lccc}
\hline \multicolumn{1}{c}{ Criterios } & A & B & C \\
\hline Breve descripción de servicios de pago & $\checkmark$ & $\checkmark$ & $\checkmark$ \\
\hline Costos de afiliación & $\checkmark$ & $\checkmark$ & \\
\hline Costos de mantenimiento & & $\checkmark$ & \\
\hline Costos de comisiones & & $\checkmark$ & \\
\hline Procedimiento claro para afiliación & $\checkmark$ & & \\
\hline
\end{tabular}




\begin{tabular}{lccc}
\hline Procedimiento de implementación & & \multicolumn{2}{c}{ Parcial } \\
\hline $\begin{array}{l}\text { Explicación sobre los requisitos de } \\
\text { afiliación }\end{array}$ & Parcial & & \\
\hline Certificado de seguridad & $\checkmark$ & $\checkmark$ & $\checkmark$ \\
\hline Videos informativos & $\checkmark$ & & \\
\hline Información de contacto & $\checkmark$ & $\checkmark$ & $\checkmark$ \\
\hline Navegación segura & $\checkmark$ & $\checkmark$ & \\
\hline Total de criterios encontrados & 7 & 7 & 3 \\
\hline
\end{tabular}

Nota: A. Payphone; B. Kushki; C. B-wise.

Fuente: Payphone (2019); Kushki (2019); B-wise (2019)

La Tabla 2, evidencia la escasa información relacionada con los requisitos, costos y procedimientos de afiliación e implementación de las soluciones FTP en Ecuador, por consiguiente se procedió a realizar entrevistas telefónicas con agentes de ventas de las empresas observadas para profundizar los datos pertinentes, lo que permitió contar con insumos necesarios en la construcción de los resultados de la investigación.

\section{MARCO CONCEPTUAL}

\section{Antecedentes de las FinTech}

Los estudios del BBVA Innovation Center (2016) señalan que el origen de las FinTech está situado en un invento de la banca francesa: el "pantelégrafo" de Giovanni Caselli, utilizado en 1865 durante las transacciones bancarias para la autenticación de firmas, años después en 1950 aparecen las primeras tarjetas crediticias, y durante 1967 el cajero automático, para en 1983 integrarse la banca en línea.

Consecuentemente, el BBVA Innovation Center (2016) manifiesta que los avances de las e-finance (finanzas electrónicas) combinados con tecnologías móviles empresariales, redes sociales, inteligencia artificial y análisis Big data, impulsaron el nacimiento de las FinTech. Por su parte, Lee \& Shin (2018) consideran que la revolución del internet originó profundos cambios en los mercados financieros mundiales desde principios de los años 90, dándose origen al modelo de la banca 
móvil, que han crecido exponencialmente a partir del año 2000 debido al gran aumento de usuarios de telefonía móvil alrededor del mundo.

En este sentido, autores como González, Sojo, Carrizo, Morales, y Espíndola (2018) señalan que las primeras empresas FinTech en Latinoamérica se identifican desde el 2014. La propuesta de valor que aparece con éstas, se basa en diseñar productos para grupos tradicionalmente desatendidos por las instituciones bancarias, haciéndolos asequibles para hogares y empresas de ingresos menores como PyMEs; a diferencia de la banca tradicional que debido a su naturaleza adversa al riesgo excluye ciertos servicios únicamente para personas o entidades con un determinado rango de ingresos.

\section{Características de las FinTech}

A diferencia de la oferta de multi-productos de la banca tradicional, la propuesta principal de toda FinTech está relacionada con un aspecto en concreto de las finanzas, su simplicidad permite que se conviertan en auténticas especialistas en el área de negocio que prefieran (Igual, 2018), entre las características distintivas aportadas por López, Langthaler, Fabian, y Mayorga (2017) e Igual (2018) se detallan las siguientes:

Tabla 3. Características de las empresas FinTech

\begin{tabular}{|c|c|}
\hline Positivas & Negativas \\
\hline $\begin{array}{l}\text { Orientadas a un aspecto de las } \\
\text { finanzas }\end{array}$ & \\
\hline $\begin{array}{l}\text { - Se apoyan fuertemente de la } \\
\text { tecnología }\end{array}$ & $\begin{array}{l}\text { - Ciber-seguridad } \\
\circ \text { Robo de información }\end{array}$ \\
\hline - Son altamente innovadoras & \\
\hline - Costos operativos bajos & \\
\hline - Procesos operativos más rápidos & \\
\hline
\end{tabular}

Fuente: López et al. (2017); Igual (2018)

- Su orientación les permite brindar una oferta vertical a la de las instituciones financieras, por lo que inclusive ha conllevado a alianzas entre ambas. 
- El apoyo tecnológico es indispensable para cubrir los problemas financieros o las necesidades no atendidas de sus clientes, principalmente utilizan plataformas, aplicaciones móviles, o dispositivos innovadores.

- Su cultura de innovación promete romper con estereotipos tradicionales de cliente vs la poderosa banca, y engloba una filosofía de relación peer to peer (persona a persona).

- Manejan bajos costos operativos, debido a que se enfocan en un determinado aspecto financiero.

- Emplea procesos operativos más veloces para conectarse con el cliente debido a su estructura sencilla.

- Entre sus riesgos se encuentra la ciber-seguridad y el robo de información, controlables siempre y cuando la empresa cumpla con parámetros de seguridad tecnológica emitidos por organismos internacionales como los del Consejo de Normas de Seguridad (Security Standards Council, PCl), es por ello que el protocolo de información (https) debe contar con un certificado de seguridad para la confidencialidad y protección de la integridad de los datos pertenecientes a los usuarios.

\section{Modelos de negocio FinTech}

Para analizar los distintos modelos de negocio FinTech, se han revisado los aportes de Hoder, Wagner, Sguerra, y Bertol (2016); Inter-American Development Bank (IDB) \& Finnovista (2017); Maldonado \& Naranjo (2017); Orueta et al. (2017); Igual (2018); González, Sojo, Carrizo, Morales, y Espíndola (2018) y Cámara de Comercio de Bogotá (2019), a fin de determinar las que reflejen mayor número de coincidencias, las semejanzas se cotejaron en la siguiente tabla comparativa: 
Tabla 4. Análisis comparativo de modelos de negocio FinTech

\begin{tabular}{|c|c|c|c|c|c|c|c|}
\hline Modelos de negocio FinTech & 1 & 2 & 3 & 4 & 5 & 6 & 7 \\
\hline Crowdfunding & $\mathrm{x}$ & $\mathrm{x}$ & $\mathrm{x}$ & $\mathrm{x}$ & & $\mathrm{x}$ & $\mathrm{x}$ \\
\hline Prestamos P2P / Lending & $\mathrm{x}$ & $\mathrm{x}$ & $\mathrm{x}$ & $\mathrm{x}$ & $\mathrm{x}$ & & \\
\hline Sistemas de pago y transferencias & $\mathrm{x}$ & $\mathrm{x}$ & $\mathrm{x}$ & $\mathrm{x}$ & $\mathrm{x}$ & & $x$ \\
\hline Gestión de finanzas personales & & $\mathrm{x}$ & $\mathrm{x}$ & & $\mathrm{x}$ & $\mathrm{x}$ & $\mathrm{x}$ \\
\hline Gestión de finanzas empresariales & & $\mathrm{x}$ & $\mathrm{x}$ & & $\mathrm{x}$ & $\mathrm{x}$ & $\mathrm{x}$ \\
\hline $\begin{array}{l}\text { Gestión de inversiones y } \\
\text { asesoramiento financiero }\end{array}$ & & $\mathrm{x}$ & $\mathrm{x}$ & $\mathrm{x}$ & $\mathrm{x}$ & $\mathrm{x}$ & $\mathrm{X}$ \\
\hline Roboadvisor & & & & & $\mathrm{x}$ & & \\
\hline Inteligencia de mercados & $\mathrm{x}$ & & & & & & \\
\hline Financiamiento de cadenas de valor & $\mathrm{x}$ & & & & & & \\
\hline Criptomonedas y monedas alternativas & & & $\mathrm{x}$ & $\mathrm{x}$ & $\mathrm{x}$ & & \\
\hline Blockchain o DTL & & & $\mathrm{x}$ & $\mathrm{x}$ & $\mathrm{x}$ & & \\
\hline $\begin{array}{l}\text { Verificación de identidad, fraudes y } \\
\text { scorings }\end{array}$ & & & $\mathrm{x}$ & & $\mathrm{x}$ & $\mathrm{x}$ & \\
\hline Big Data y modelos predictivos & & & & $\mathrm{x}$ & $\mathrm{x}$ & & \\
\hline $\begin{array}{l}\text { Inteligencia de mercados/ Mercado de } \\
\text { valores }\end{array}$ & $\mathrm{x}$ & $\mathrm{x}$ & & & & $\mathrm{x}$ & \\
\hline Seguros & & $\mathrm{x}$ & & & $\mathrm{x}$ & $\mathrm{x}$ & \\
\hline $\begin{array}{l}\text { Aplicaciones para instituciones } \\
\text { financieras }\end{array}$ & & $x$ & & & $x$ & $x$ & $x$ \\
\hline $\begin{array}{l}\text { Banca digital } \\
\text { Educación financiera }\end{array}$ & & $x$ & & $x$ & $x$ & $x$ & $x$ \\
\hline
\end{tabular}

Nota: 1. Hoder et al. (2016); 2. Inter-American Development Bank (IDB) \& Finnovista (2017); 3. Maldonado \& Naranjo (2017); 4. Orueta et al. (2017); 5. Igual (2018); 6. González, Sojo, Carrizo, Morales, y Espíndola (2018); 7. Cámara de Comercio de Bogotá (2019).

El análisis refleja coincidencias principalmente en los modelos de: Crowdfunding; Préstamos P2P; Sistemas de pagos y transferencias, Gestión de finanzas personales, empresariales, y las de inversiones y asesoramiento financiero; Aplicaciones para instituciones financieras; y Banca digital, a su vez estas clasificaciones pueden contener sub-clasificaciones englobándose otra tipología no tomada en cuenta como principal. A continuación se explica cada una, a partir de la literatura consultada: 
- Crowdfunding: Involucra una red de personas que abarca promotores de proyectos que buscan inversores u oferentes, su mecanismo consiste en asociar al creador de un proyecto (persona o empresa de ingresos menores como las PyMEs) con potenciales inversionistas que generalmente buscan valores o participación social a cambio, aunque también existen las que no involucran recompensas monetarias, en este grupo se hallan las plataformas para donaciones como GoFundMe.

- Préstamos Peer to Peer (P2P): Préstamos de persona a persona (P2P) es un modelo que utiliza una estructura que permite el contacto entre prestamistas y prestatarios, permite que las personas o los negocios tengan la facilidad de pedir 0 prestar valores económicos con bajos intereses. No involucra la transacción en sí, solo el contacto.

- Sistemas de pagos y transferencias: Este modelo es el de mayor crecimiento en la región latinoamericana, consiste en servicios que agilitan la recepción de pagos, transferencias internacionales y remesas; a través de una plataforma o terminal de venta.

- Gestión de finanzas personales: Brindan apoyo en la gestión inteligente del dinero de sus usuarios, abarca las plataformas de ahorro a través de la gestión de gastos como 1Money, Gestión de deudas, Plataformas de comparación de productos financieros.

- Gestión de finanzas empresariales: Involucran un modelo de negocio de apoyo al crecimiento empresarial, a través de herramientas de gestión como facturación electrónica, cobranzas, contabilidad digital, soluciones de análisis de datos. Safi es una de las FinTech ecuatorianas destacada en este ámbito.

- Gestión de inversiones y asesoramiento financiero: Plataformas robot de asesoramiento financiero a bajo costo, funcionan bajo inteligencia artificial o algoritmos que involucran alternativas de inversión en base a características y preferencias de clientes. 
- Aplicaciones para instituciones financieras: A pesar de que en un inicio las FinTech se consideraban competencia de la banca tradicional hoy en día trabajan como aliadas, principalmente apoyan en servicios que usualmente no cubrían debido al costo o su dificultad de alcance.

- Banca digital: Un modelo que presenta distribución digital de múltiples productos financieros, con licencia propia o utilizando la de un tercero (institución financiera tradicional) a fin de que sus clientes ahorren tiempo. A pesar de que en el continente asiático ha tenido gran aceptación, en Latinoamérica aún está en etapa de consolidación.

En el marco de las observaciones anteriores, se presentan los principales modelos FinTech en Latinoamérica y Ecuador a partir de los estudios realizados por el InterAmerican Development Bank (IDB) \& Finnovista (2017), y Finnovista (2017):

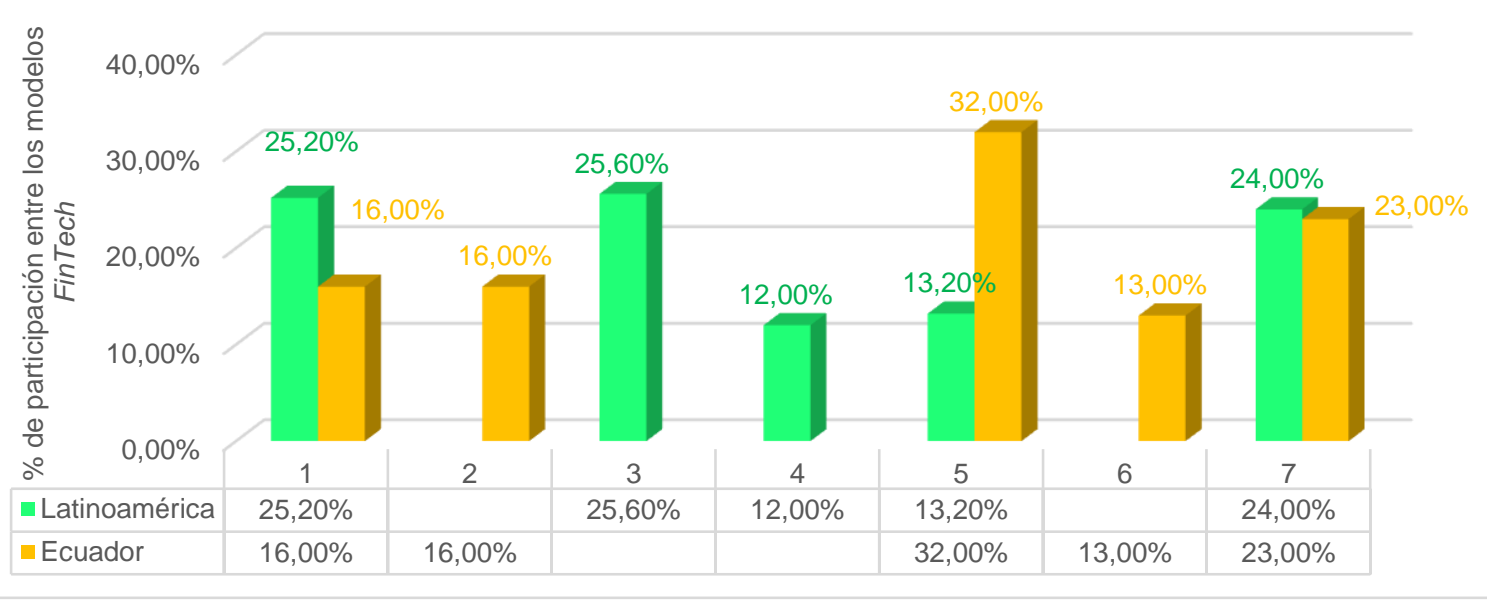

Gráfico 1. Principales modelos FinTech en Latinoamérica y Ecuador

Nota: 1. Sistemas de pagos y transferencias; 2. Aplicaciones para instituciones financieras; 3. Préstamos Peer to Peer (P2P); 4. Identidad de fraudes; 5. Gestión de finanzas empresariales; 6. Crowdfunding; 7. Otros (inferiores al 10\%).

Fuente: Inter-American Development Bank (IDB) \& Finnovista , 2017); Finnovista (2017) 
La gráfica 1, compara los principales modelos FinTech de Latinoamérica y Ecuador; se observa que a nivel nacional se destaca el modelo de Gestión de Finanzas Empresariales, mientras que en el panorama general Latinoamericano sobresale el Sistema de pagos y transferencias. Estudios del Inter-American Development Bank (IDB) \& Finnovista (2017), han permitido vislumbrar una oportunidad de desarrollo en el modelo de sistema de pagos, ya que se presume ganará mayor participación en el futuro, al igual que en otros países de la región como Colombia (27\%), Brasil (23\%), Argentina (22\%) y México (20\%), en donde se mantiene liderando el mercado FinTech.

\section{ECOSISTEMA FINTECH}

Para comprender la dinámica del ecosistema FinTech, se analizaron las coincidencias en los elementos propuestos por González et al. (2018) y Lee \& Shin (2018) quienes sugieren una relación entre los siguientes elementos:

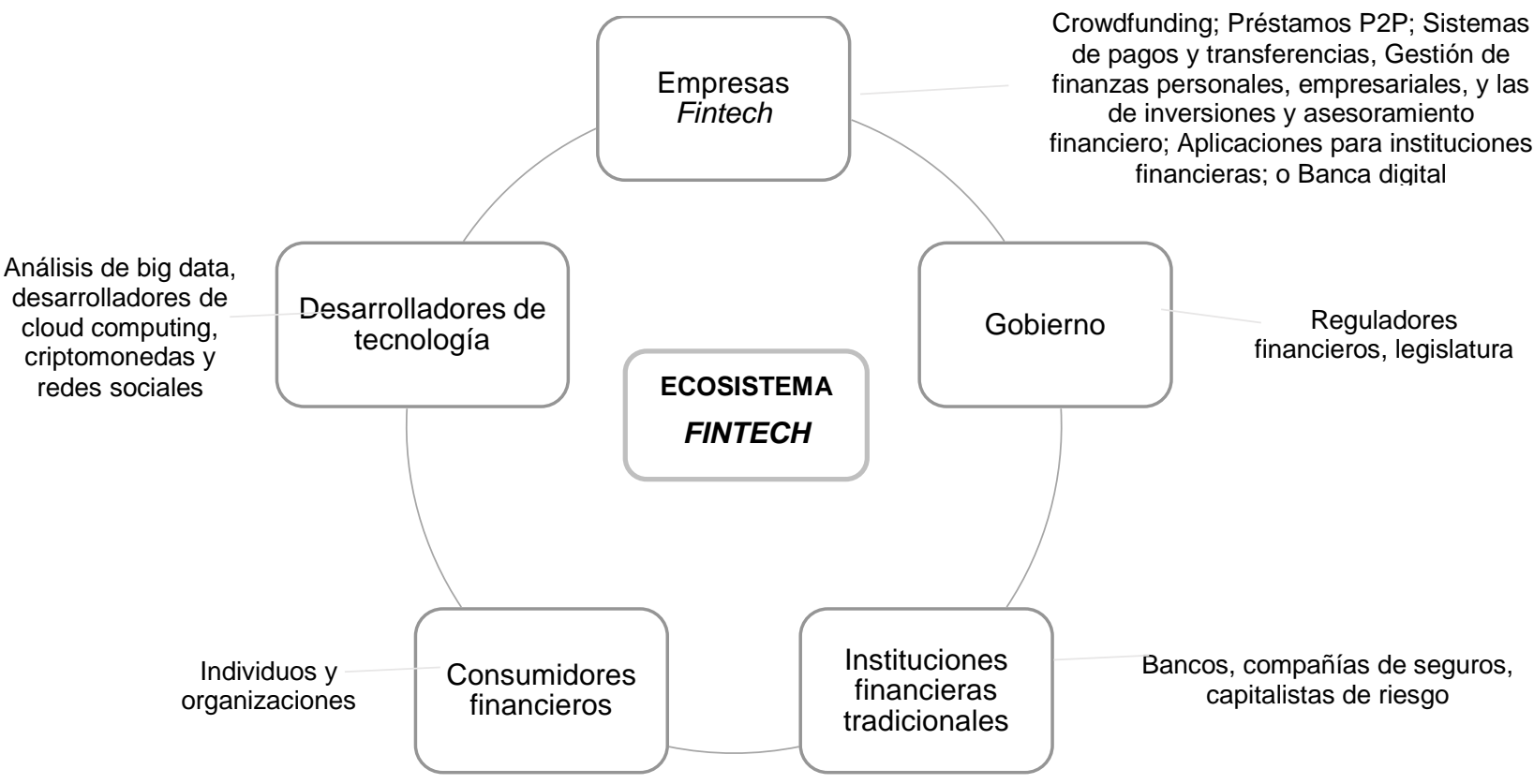

Figura 1. Elementos del ecosistema FinTech

Fuente: González et al. (2018); Lee \& Shin (2018) 
- Empresas Fintech, actualmente esta industria cuenta con significativa presencia en los continentes Asiático y Europeo, mientras se abre paso a lo largo de Latinoamerica, estudios pertinentes como los del Inter-American Development Bank (IDB) \& Finnovista (2017) aseguran un desarrollo vertiginoso para las FinTech y evidencian el mercado potencial para éstos modelos de negocio; en este sentido, el panorama nacional de acuerdo con el Radar de Finnovista (2017) refleja una oportunidad de desarrollo principalmente en los procesos de acceso al pago en línea, al facilitar a las PyMEs un nuevo canal de pago para sus clientes.

- Desarrolladores de tecnología, el papel que desempeñan es fundamental para llevar a cabo las plataformas en línea y móviles de servicios FinTech; de manera que Lee \& Shin (2018) consideran que su participación ha logrado que empresas con pocos recursos logren acceder a servicios financiero-tecnológicos a bajos costos, originándose plazas laborales emergentes para este sector.

- Instituciones financieras tradicionales, Chishti \& Barberis, (2017) expresan que aunque a lo largo de la historia han sido los grandes bancos los responsables de las innovaciones financieras, la realidad actual ha permitido que nuevos emprendimientos de tipo FinTech se plantearan como competidores de la banca tradicional, sin embargo Igual (2018) señala que en varios casos Banca y FinTech trabajan de manera colaborativa a fin de brindar un mejor servicio al cliente final.

- Consumidores financieros, uno de los retos que representa ésta disruptiva tecnológica es la acogida de usuarios de sus servicios digitales, en principio uno de los canales que más se estimularían sería el móvil. La ubicuidad de los smartphones suplantará a futuro las transacciones físicas por virtuales, a una fracción de costo. Los consumidores podrán elegir entre los servicios de la banca y los de una variedad de empresas FinTech emergentes, así lo señala González et al. (2018).

- Gobierno, en materia de regulación de las FinTech, Hoder et al.(2016) contempla la necesidad de adoptar normativas sobre la protección y seguridad en las transacciones electrónicas y promover diálogos constructivos entre actores del 
sector, esto cimentará las bases de la integridad del sistema de pagos, tasas, administración de riesgos y demás parámetros implicados en la regulación como los señalados por la Cámara de Comercio de Bogotá (2019) y otros como la protección del usuario y la seguridad cibernética. A lo largo de la región latinoamericana, México ha sido el único país en contar con una "Ley FinTech" para normar los principios bajo los cuales deben desempeñarse dichas empresas; por otro lado en el caso de Ecuador, tras 113 años de vigencia del Código de Comercio dictado en 1906 se han realizado nuevas reformas, así lo corroboran El Universo (2019) y Rodríguez (2019), no obstante aún no se desarrolla una normativa regulatoria específica para empresas de tipo FinTech.

Para González et al. (2018), los elementos del ecosistema conviven de manera asociativa, en un entorno colaborativo que fomenta el desarrollo económico y la innovación de la industria financiera. Esta dinámica del ecosistema se entiende de la siguiente manera: principalmente enlaza las empresas FinTech con desarrolladores tecnológicos que con el apoyo de las instituciones financieras tradicionales desarrollan iniciativas de servicios tecnológico-financieros innovadores y cubren brechas de clientes (especialmente se enfocan en las PyMEs), estos consumidores financieros cada día vislumbran nuevas alternativas accesibles y seguras para cubrir sus requerimientos y fomentar su crecimiento, finalmente el gobierno es quien tiene como responsabilidad normar la relación entre los elementos del ecosistema.

\section{RESULTADOS}

\section{INCLUSIÓN FINANCIERA DIGITAL - MEDIOS DE PAGOS}

Acorde con el objetivo de investigación, el presente estudio asume un modelo FinTech específico: Sistemas de pagos, uno de principales en Latinoamérica y Ecuador de acuerdo con estudios internacionales del Inter-American Development Bank (IDB) \& Finnovista (2017), el cual lo considera como una oportunidad de apoyo para empresas 
en desarrollo como PyMEs, que a pesar de no contar con un departamento tecnológico para diseñar plataformas de pago propias, hoy en día gracias a éstas iniciativas pueden acceder a un nuevo canal que facilita tales procesos a sus clientes. En Ecuador, se identificaron tres empresas especializadas en sistemas de pago: Payphone, Kushki y Bwise, entre las cuales se realiza el siguiente análisis comparativo:

Tabla 5. Análisis comparativo de las FinTech ecuatorianas, especializadas en sistemas de pago

\begin{tabular}{|c|c|c|}
\hline Payphone & Kushki & B-wise \\
\hline $\begin{array}{l}\text { Plataforma de pagos } \\
\text { virtual }\end{array}$ & Plugin de pagos virtual & $\begin{array}{l}\text { Herramienta de pagos } \\
\text { "PayMovil" }\end{array}$ \\
\hline $\begin{array}{c}\text { Utiliza una aplicación } \\
\text { móvil para el proceso, } \\
\text { también puede integrarse } \\
\text { directamente en la página } \\
\text { web del cliente }\end{array}$ & $\begin{array}{l}\text { Funciona a través de } \\
\text { integración con la página } \\
\text { web del cliente }\end{array}$ & $\begin{array}{l}\text { Utiliza un dispositivo } \\
\text { electrónico con conexión al } \\
\text { móvil vía bluetooth. }\end{array}$ \\
\hline Transacción virtual & Transacción virtual & Transacción física \\
\hline \begin{tabular}{ll} 
& \multicolumn{1}{c}{ Requiere: } \\
$\circ$ & Conexión a internet \\
$\circ$ & Afiliación del cliente a \\
& la plataforma.
\end{tabular} & $\begin{array}{l}\quad \text { Requiere: } \\
\circ \quad \text { Conexión a internet } \\
\text { Cumplir un monto de } \\
\text { comisiones de mínimo } \\
\$ 100\end{array}$ & \begin{tabular}{ll} 
& \multicolumn{1}{c}{ Requiere: } \\
$\circ$ & Conexión a internet \\
$\circ$ & Un dispositivo móvil con \\
sistema Android o IOS
\end{tabular} \\
\hline Recibe pagos a distancia. & Recibe pagos a distancia. & $\begin{array}{c}\text { No recibe pagos a distancia, } \\
\text { tiene versatilidad para } \\
\text { trasladar el dispositivo de } \\
\text { cobro hacia el cliente. }\end{array}$ \\
\hline $\begin{array}{l}\text { Ámbito de aplicación: } \\
\text { Transacciones } \\
\text { comerciales empresa - } \\
\text { cliente }\end{array}$ & $\begin{array}{l}\text { Ámbito de aplicación: } \\
\text { Transacciones } \\
\text { comerciales empresa - } \\
\text { cliente }\end{array}$ & $\begin{array}{l}\text { Ámbito de aplicación: } \\
\text { Transacciones comerciales } \\
\text { empresa - cliente }\end{array}$ \\
\hline
\end{tabular}


Costos:

- Afiliación gratuita

- Cobro de afiliación mensual: 0

- Comisión por transacción: 0

- Comisión de cobro con tarjeta de crédito: $4,58 \%$

- Comisión de cobro con tarjeta de débito: $2 \%$

Tarjetas aceptadas:

- Crédito: Visa o Mastercard

- Débito: Banco del Austro, Guayaquil, Pichincha y Produbanco
Costos:

Afiliación gratuita

- Cobro mínimo de afiliación mensual: $\$ 100,00$

- Comisión por transacción: $1,5 \%+$ $\$ 0,50+$ iva

- Comisión de cobro con tarjeta de crédito: de 4 a $13 \%$

- Comisión de cobro con tarjeta de débito: $4 \%$
Costos:

- Afiliación: \$128,80 (incluye dispositivo)

- Cobro de mantenimiento mensual: $\$ 8,95$

- Comisión por transacción: 0

- Comisión de cobro con tarjeta de crédito: $4,5 \%$ en adelante

- Comisión de cobro con tarjeta de débito: 2,24\%
Tarjetas aceptadas:

- Crédito: Visa, Mastercard y American

Express

- Débito: Banco del Austro, Guayaquil, Pichincha y Produbanco
Tarjetas aceptadas:

- Crédito: Visa, Mastercard, Diners Club, Visa Electron, Discover, Maestro, y American Express

- Débito: Banco Guayaquil, Pacífico, y Pichincha.

Certificado de seguridad de datos PCI DSS 3.2

Certificado de seguridad de datos PCI DSS nivel 1

Certificado de seguridad de datos $\mathrm{PCl}$

Grado de confiabilidad Grado de confiabilidad $4,7 / 5$

*Basado en 88 opiniones de sus usuarios.
$2,4 / 5$

*Basado en 12 opiniones de sus usuarios.
Grado de confiabilidad 2,2/5

*Basado en 8 opiniones de sus usuarios.

Fuente: Payphone (2019); Kushki (2019); B-wise (2019)

La Tabla 5, refleja las posibilidades de afiliación a las que podrían acceder empresas en desarrollo como PyMEs; el hecho de optar por un canal de pagos apoyado en la tecnología brindaría una oportunidad de crecimiento y expansión al negocio. A primera vista, se destaca el servicio de la empresa Payphone, debido a su nivel de confiabilidad y costos accesibles para negocios de ingresos menores que busquen expansión, sin embargo como limitantes se halla la baja aceptabilidad de tarjetas de crédito, no 
obstante, si se aplicara para el área de comercio minorista, por lo general los pagos se harían con tarjeta de débito bancario por lo que no representaría un mayor problema.

\section{CONSTRUCCIÓN DEL PROCEDIMIENTO METODOLÓGICO}

Para Chishti \& Barberis (2017), incursionar con tecnologías, se ha convertido en un factor clave para desbloquear mercados, es por ello que implementar una solución FTP, promueve una ventaja competitiva para las empresas que deseen aplicarlas. Para alcanzar el fin propuesto, tras el análisis de las actividades necesarias para implementar una solución de pagos en PyMEs ecuatorianas, se propone el siguiente procedimiento metodológico que contempla ocho fases:

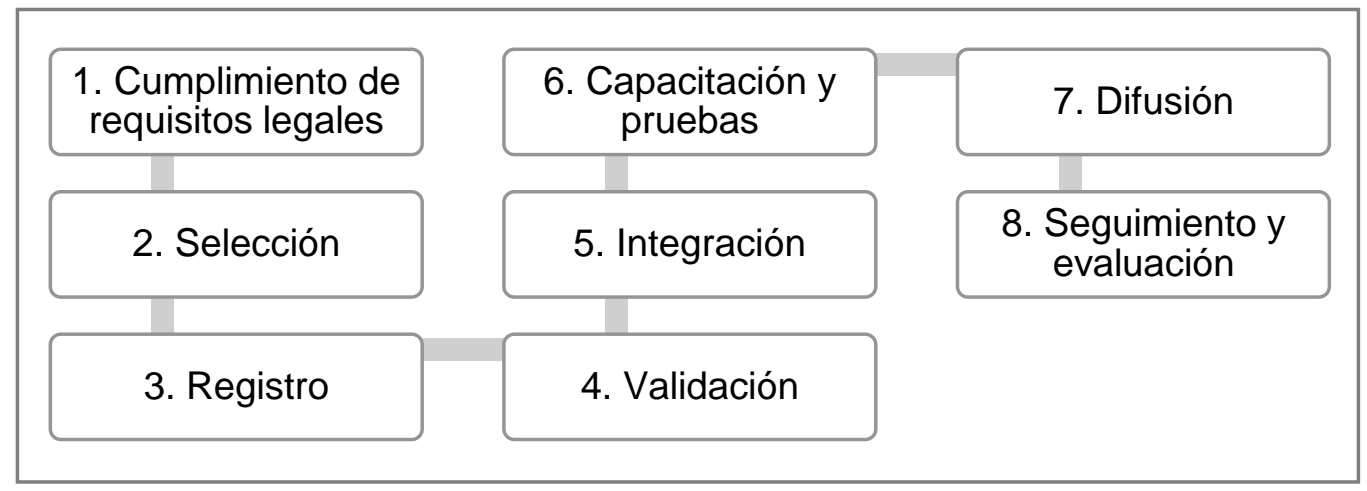

Figura 2. Procedimiento metodológico para implementación de soluciones de pago FinTech para PyMEs

Cabe mencionar que las fases propuestas están sujetas a modificación de acuerdo con la realidad de la empresa que las aplique, y el avance de las normativas y regulaciones de tipo FinTech de cada país.

Fase 1: Cumplimiento de requisitos legales, la empresa aplicante deberá estar legalmente constituida y contar con la siguiente documentación:

- Registro de contribuyente ante la autoridad tributaria para desarrollar una actividad económica formal, en el caso ecuatoriano este puede ser: el Registro 
Único de Contribuyente (RUC) o Régimen Impositivo Simplificado Ecuatoriano (RISE)

- Documentos de identidad del representante legal

- Recibo de pago de un servicio básico (luz, agua, teléfono)

- Escritura de constitución de la empresa

- Última declaración de impuesto (Impuesto al Valor Agregado IVA)

- Certificación de cuenta bancaria

- Balance contable del año anterior a la afiliación

Fase 2: Selección, desarrolla el proceso de selección del proveedor FTP.

* Revisar las alternativas de proveedores, en el caso de Ecuador se puede tomar de apoyo la Tabla 5.

- Desarrollar un método de evaluación de alternativas, se puede realizar a través de las siguientes matrices:

- Matriz de perfil competitivo (MPC), de acuerdo con Fred (2008), en esta matriz se asigna un peso ponderado al criterio de acuerdo a su relevancia en la decisión final, según la opinión de expertos de la empresa; luego se califican los criterios por cada proveedor basándose en una escala del 1 al 4 , donde 4=fortaleza principal, $3=$ fortaleza menor, $2=$ debilidad menor, y $1=$ debilidad principal.

Tabla 6. Ejemplo de ponderación de criterios

\begin{tabular}{|c|c|c|c|c|c|c|c|}
\hline \multirow{2}{*}{$\begin{array}{c}\text { Criterios/Factores } \\
\text { clave }\end{array}$} & \multirow[t]{2}{*}{ Peso } & \multicolumn{3}{|c|}{$\begin{array}{c}\text { Evaluación } \\
\text { individual }\end{array}$} & \multicolumn{3}{|c|}{$\begin{array}{l}\text { Evaluación } \\
\text { ponderada }\end{array}$} \\
\hline & & A & B & C & A & B & C \\
\hline \multirow{2}{*}{$\begin{array}{l}\text { Costo de afiliación } \\
\text { Costo de } \\
\text { mantenimiento }\end{array}$} & $10 \%$ & 4 & 4 & 1 & 0,40 & 0,40 & 0,10 \\
\hline & $10 \%$ & 4 & 1 & 2 & 0,40 & 0,10 & 0,20 \\
\hline \multirow{3}{*}{$\begin{array}{l}\text { Costo de comisiones } \\
\text { Aceptabilidad de } \\
\text { tarjetas } \\
\text { Sequridad }\end{array}$} & $20 \%$ & 4 & 2 & 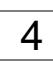 & 0,80 & 0,40 & 0,80 \\
\hline & $30 \%$ & 2 & 3 & 4 & 0,60 & 0,90 & 1,20 \\
\hline & $15 \%$ & 4 & 2 & 4 & 0,60 & 0,30 & 0,60 \\
\hline
\end{tabular}




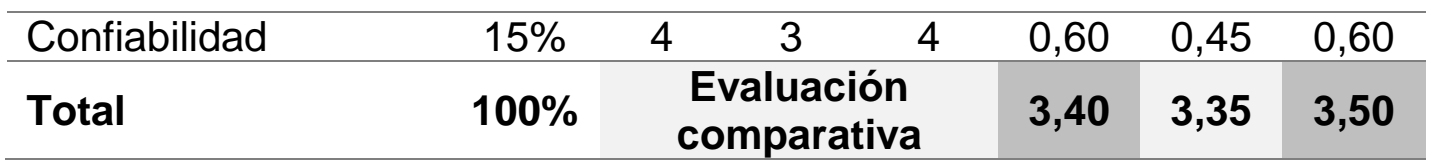

Nota: A. Payphone; B. Kushki; C. B-wise. Los valores del peso por criterio se han determinado a criterio del autor a modo de ejemplo.

Fuente: adaptado de Lee y Larry (2000); Fred (2008); Cantú (2011)

En la Tabla anterior, al tomar en cuenta seis criterios relacionados con costos, aceptabilidad, seguridad y confiabilidad, la alternativa que destaca en puntuación es C., sin embargo la diferencia con la que supera a la alternativa A. es mínima $(0,10)$, por lo que dependerá de la empresa tomar dichos resultados como válidos o repetir una ponderación con más criterios entre estas dos alternativas.

- Matriz de priorización (MP), de acuerdo con Betancourt (2018); Aiteco Consultores S.L. (2019); y, Asociación Española para la Calidad (AEC) (2019), busca objetivar la selección de una alternativa a través de una evaluación entre criterios y alternativas, el proceso se detalla a continuación:

1. Determinar las alternativas disponibles, y los criterios que regirán la evaluación, la variedad de criterios definirán un mejor resultado en la matriz.

Tabla 7. Alternativas y criterios de evaluación

\begin{tabular}{|c|c|c|c|}
\hline \multicolumn{2}{|c|}{$\begin{array}{c}\text { Opciones/ } \\
\text { Alternativas }\end{array}$} & \multicolumn{2}{|r|}{ Criterios } \\
\hline A & Payphone & 1 & Costo de la afiliación \\
\hline B & Kushki & 2 & Costo de mantenimiento \\
\hline C & B-wise & 3 & Costo de comisiones \\
\hline & & 4 & Aceptabilidad de tarjetas de crédito \\
\hline & & 5 & Aceptabilidad de tarjetas de débito \\
\hline & & 6 & Confiabilidad \\
\hline & & 7 & Facilidad de integración \\
\hline & & 8 & Facilidad de uso \\
\hline & & 9 & Facilidad de cambios y configuraciones \\
\hline & & 10 & Visualización de paneles de resultados \\
\hline
\end{tabular}


2. Determinar la importancia relativa de los criterios en la tabla de Ponderación de criterios, contrastando columna sobre fila, la dinámica se establece a partir de la siguiente escala de evaluación, propuesta por Betancourt (2018):

Tabla 8. Escala de evaluación de criterios

\begin{tabular}{clc}
\hline Valor & \multicolumn{1}{c}{ Detalle } & $\begin{array}{c}\text { Valor } \\
\text { recíproco }\end{array}$ \\
\hline 10 & CC es mucho más importante que CF & 0,1 \\
5 & CC es más importante que el CF & 0,2 \\
1 & CC y CF son igual de importantes. & 1 \\
0,2 & CC es menos importante que CF & 5 \\
0,1 & CC es mucho menos importante que CF & 10 \\
\hline
\end{tabular}

Nota: $\mathrm{CC}=$ Criterio de columna; $\mathrm{CF}=$ Criterio de fila.

Fuente: Betancourt (2018)

Tabla 9. Ponderación de criterios

\begin{tabular}{ccccccccccccc}
\hline Criterios & $\mathbf{1}$ & $\mathbf{2}$ & $\mathbf{3}$ & $\mathbf{4}$ & $\mathbf{5}$ & $\mathbf{6}$ & $\mathbf{7}$ & $\mathbf{8}$ & $\mathbf{9}$ & $\mathbf{1 0}$ & Total $\begin{array}{c}\text { Peso } \\
\begin{array}{c}\text { ponderado } \\
\text { definido } \\
\text { (PPD) }\end{array}\end{array}$ \\
\hline $\mathbf{1}$ & & 1 & 0,1 & 0,2 & 0,2 & 0,1 & 5 & 0,2 & 0,2 & 0,1 & 7,10 & 0,03 \\
$\mathbf{2}$ & 1 & & 0,1 & 0,2 & 0,2 & 0,1 & 0,2 & 0,2 & 1 & 1 & 4,00 & 0,02 \\
$\mathbf{3}$ & 10 & 10 & & 0,2 & 0,2 & 0,1 & 0,2 & 0,2 & 0,1 & 0,2 & 21,20 & 0,09 \\
$\mathbf{4}$ & 5 & 5 & 5 & & 1 & 1 & 0,2 & 0,2 & 0,2 & 1 & 18,60 & 0,08 \\
$\mathbf{5}$ & 5 & 5 & 5 & 1 & & 1 & 0,2 & 0,2 & 0,2 & 1 & 18,60 & 0,08 \\
$\mathbf{6}$ & 10 & 10 & 10 & 1 & 1 & & 5 & 5 & 1 & 5 & 48,00 & 0,20 \\
$\mathbf{7}$ & 0,2 & 5 & 5 & 5 & 5 & 0,2 & & 0,2 & 1 & 1 & 22,60 & 0,09 \\
$\mathbf{8}$ & 5 & 5 & 5 & 5 & 5 & 0,2 & 5 & & 5 & 1 & 36,20 & 0,15 \\
$\mathbf{9}$ & 5 & 1 & 1 & 5 & 5 & 1 & 1 & 0,2 & & 0,1 & 19,30 & 0,08 \\
$\mathbf{1 0}$ & 10 & 10 & 5 & 1 & 1 & 0,2 & 5 & 1 & 10 & & 43,20 & 0,18 \\
\hline & & & & & & & & & & & $\mathbf{2 3 8 , 8 0}$ & $\mathbf{1 , 0 0}$ \\
\hline
\end{tabular}


3. Realizar la Calificación de Alternativas por Criterio (CAC), donde se asigna una calificación individual por cada alternativa en base a los 10 criterios detallados en la Tabla 7.

Tabla 10. Calificación de alternativa por criterio

\begin{tabular}{cccccc}
\hline Criterio 1 & A & B & C & Total & $\begin{array}{c}\text { Peso } \\
\text { relativo }\end{array}$ \\
\hline A & & 1 & 10 & 11 & 0,5 \\
\hline B & 1 & & 10 & 11 & 0,5 \\
\hline C & 0,1 & 0,1 & & 0,2 & 0,1 \\
\hline
\end{tabular}

4. Construir el Consolidado Total de Calificaciones de Alternativas por Criterio (CTCAC)

Tabla 11. Consolidado Total de Calificación de Alternativas por Criterio

\begin{tabular}{ccccccccccc}
\hline \multirow{2}{*}{ Alternativas } & \multicolumn{10}{c}{ Criterios } \\
\cline { 2 - 10 } & 1 & 2 & 3 & 4 & 5 & 6 & 7 & 8 & 9 & 10 \\
A & 0,50 & 0,73 & 0,59 & 0,03 & 0,33 & 0,65 & 0,65 & 0,33 & 0,48 & 0,33 \\
B & 0,50 & 0,01 & 0,01 & 0,33 & 0,33 & 0,13 & 0,22 & 0,33 & 0,48 & 0,33 \\
C & 0,01 & 0,25 & 0,40 & 0,64 & 0,33 & 0,22 & 0,13 & 0,33 & 0,03 & 0,33 \\
\hline
\end{tabular}

5. Determinar la Calificación Total, tras multiplicar cada valor de la Tabla 11. por los PPD individuales de la Tabla 9.

Tabla 12. Calificación total: alternativa por criterio ponderado

\begin{tabular}{cccccccccccc}
\hline \multirow{2}{*}{ Alternativas } & \multicolumn{1}{c}{ Criterios } & \multicolumn{1}{c}{ Total } \\
\cline { 2 - 12 }$n$ & 1 & 2 & 3 & 4 & 5 & 6 & 7 & 8 & 9 & 10 & \\
\hline A & 0,015 & 0,012 & 0,052 & 0,002 & 0,026 & 0,131 & 0,062 & 0,051 & 0,039 & 0,060 & $\mathbf{0 , 4 5}$ \\
B & 0,015 & 0,000 & 0,001 & 0,026 & 0,026 & 0,026 & 0,021 & 0,051 & 0,039 & 0,060 & 0,26 \\
C & 0,000 & 0,004 & 0,036 & 0,050 & 0,026 & 0,044 & 0,012 & 0,051 & 0,003 & 0,060 & 0,29 \\
\hline & & & & & & & & & & & $\mathbf{1 , 0 0}$
\end{tabular}


Como resultado final, en base a los PPD de la Tabla 9., con 0,19 y 0,16 de diferencia respectivamente, la mejor de las tres alternativas analizadas es $\mathrm{A}$, no obstante se recalca que los PPD fluctúan de acuerdo al juicio del grupo de trabajo encargado de la evaluación.

Fase 3: Registro, las empresas proveedoras cuentan con formularios de registro propios en sus sitios web o a través de correo electrónico, donde solicitan datos relacionados con la identificación del aplicante, su localización, tipo de negocio, ingresos de ventas, encuestas de datos de seguridad PCl, integración web, entre otros.

- Llenar el formulario de registro

APLICACIÓN PARA CUENTA KUSHKI - ECUADOR

Gracias por tu interés en Kushikit Para poder servirte pronto, vamos a necesitar que completes este formulario

\section{INFORMACIÓN DEL APLICANTE}

Número de RUC .

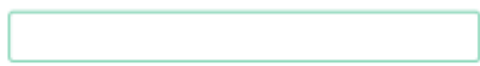

Tipo de Personeria. *

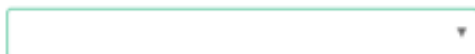

Nombre de Representante Legal. *

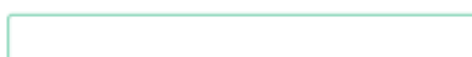

Razón social *

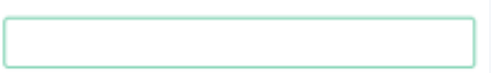

Nombre Comercial

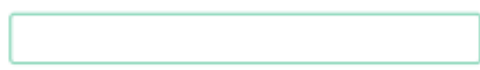

Correo Electrónico Representante Legal *

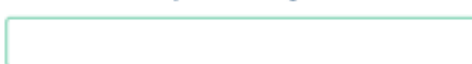

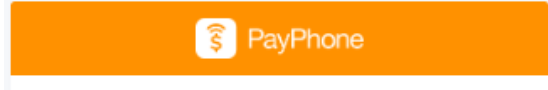

Registro

Por favor, selecciona el país y escribe tu código

único de compañía.

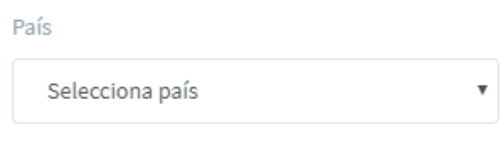

Ruc/Nit

\section{Verificar}

¿Ya tiene una cuenta?

Iniciar sesión

Figura 3. Formulario de registro en plataformas FinTech de pago ecuatorianas Fuente: Payphone (2019); Kushki (2019)

- Aceptar los términos y condiciones

- Adjuntar documentos previstos en la Fase 1, ya sea por medio de la página de afiliación de la empresa, e-mail o envío por correo postal. 
Fase 4: Validación, tras el envío de documentación, el proveedor de soluciones de pagos, inicia un proceso de revisión y validación de los datos de la empresa aplicante a fin de asegurar su legalidad y funcionamiento, posteriormente, se procede con la firma de contrato. El tiempo de validación generalmente suele ser de aproximadamente 7 días laborables, tras la validación se apertura el acceso a informes de ventas, configuración de datos, y/o validación de transacciones.

Fase 5: Integración, dependerá del proveedor FinTech solicitado, ya que manejan diferentes procesos para ello; en el caso de Kushki, se requiere necesariamente el trabajo de programadores para la integración con el sistema web de la empresa, mientras que otros como Payphone utilizan una plataforma propia y no necesariamente requieren la integración a una web externa, aunque si cuentan con el servicio de integración externa; por otro lado B-wise, requiere una integración física por medio de un pequeño dispositivo conectado por Bluetooth a un móvil con acceso internet.
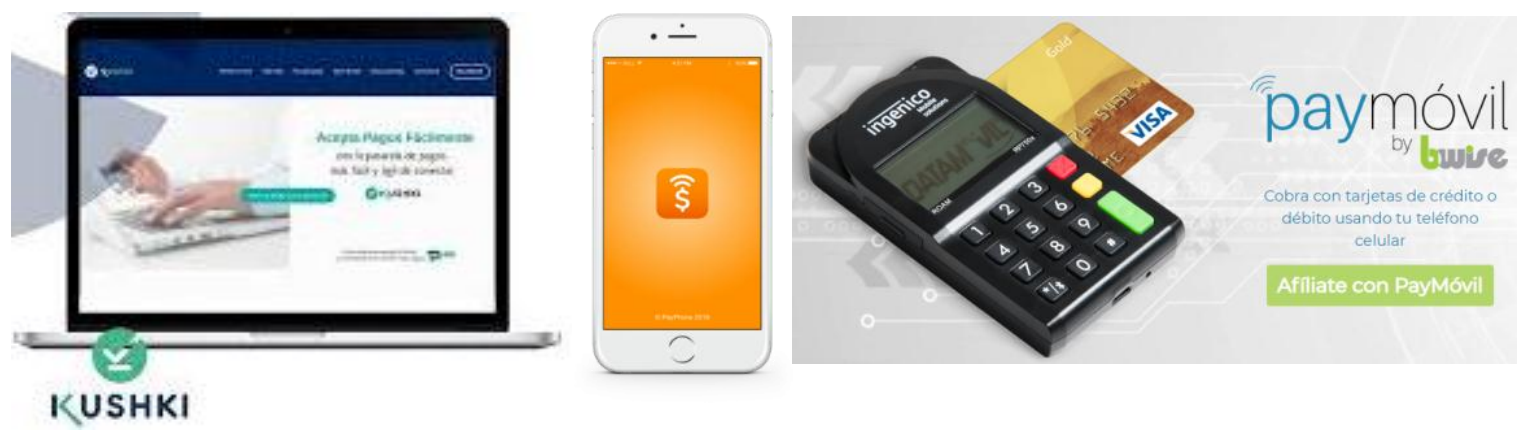

Figura 4. Integración de sistemas de pago

Fuente: Payphone (2019); Kushki (2019); B-wise (2019)

La innovación empresarial que supone la integración de una solución FTP fomentaría una ventaja competitiva para la empresa que la integre, para Martínez y El Kadi (2019) resulta crucial desarrollar ventaja en mercados altamente competitivos como en el caso de las PyMEs. 
Fase 6: Capacitación y pruebas, capacitar al personal acerca del nuevo canal de pagos, es parte fundamental de la implementación, y está bajo responsabilidad de los proveedores, algunos como B-wise lo realizan por medio de visitas y mantenimientos mensuales al equipo, y otros como Kushki y Payphone lo hacen por medio de video tutoriales en sus redes y sitios web. Se sugiere también difundir los tutoriales de pago con los clientes (Fase 7), a fin de familiarizarlos con el uso de la nueva herramienta.

Fase 7: Difusión, consiste en desarrollar el plan de difusión del nuevo canal de pagos para los clientes, en este sentido Urueta (2019) resalta la contribución de las tecnologías para la difusión de información, al considerarlas como una herramienta que promueve la comunicación eficiente; en ese grupo se encuentran los medios digitales como redes sociales. Proveedores como Payphone apoyan en la fase difusión en sus páginas oficiales (Figura 5); sin embargo, dependerá de los directores de marketing de cada empresa analizar las vías de difusión más pertinentes para ello.
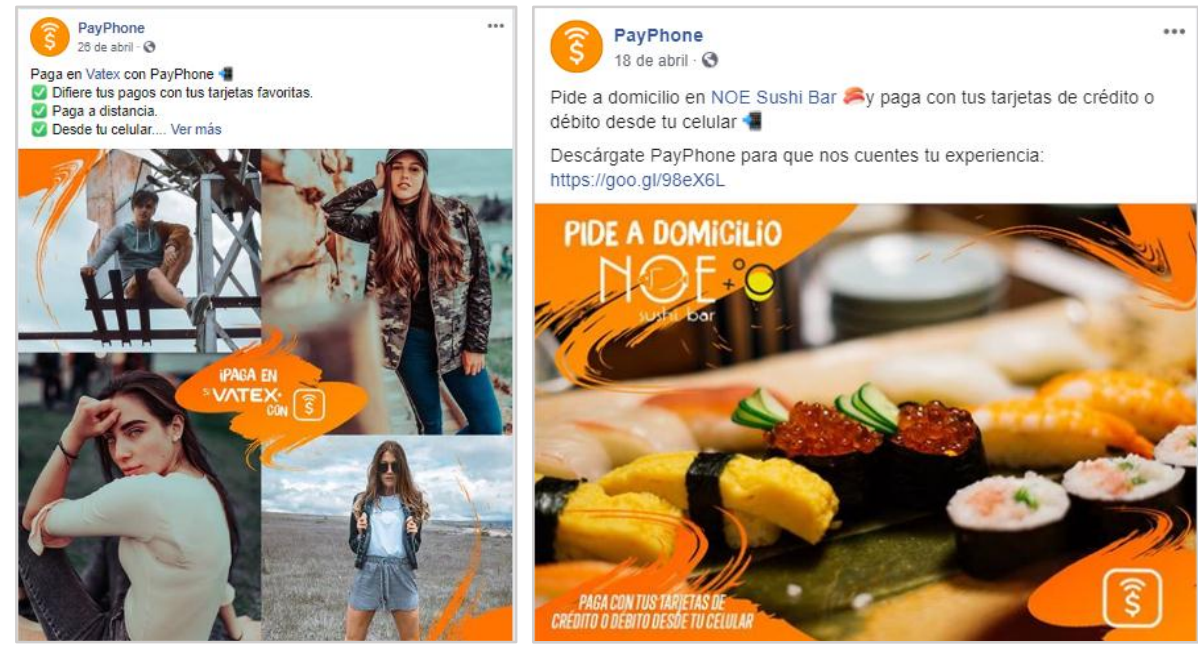

Figura 5. Difusión en redes sociales

Fuente: Payphone (2019)

Fase 8: Seguimiento y evaluación, definir indicadores permitirá sondear la eficiencia de la nueva solución de pagos implementada durante un plazo determinado. Esta es la 
fase clave que permitirá visualizar los resultados del nuevo canal de pago, la creación de los indicadores dependerá de la empresa, a continuación se detalla un ejemplo:

Tabla 13. Ejemplo de ficha del indicador Transacciones en línea

\begin{tabular}{|c|c|c|}
\hline $\begin{array}{l}\text { Nombre del } \\
\text { indicador }\end{array}$ & $\begin{array}{l}\text { Objetivo del } \\
\text { indicador }\end{array}$ & Fórmula del indicador \\
\hline $\begin{array}{l}\text { Transacciones } \\
\text { en línea }\end{array}$ & $\begin{array}{l}\text { Medir el porcentaje } \\
\text { de transacciones } \\
\text { realizadas en línea }\end{array}$ & $\frac{\text { Transacciones en línea }}{\text { Total de transacciones }} \times 100$ \\
\hline Meta & Responsable & Frecuencia de reporte \\
\hline $\begin{array}{c}50 \\
\text { transacciones }\end{array}$ & Vendedor 1 & Mensual \\
\hline
\end{tabular}

Nota: Los valores se han fijado a criterio del autor para ejemplificar

Fuente: Villagra (2016)

\section{CONCLUSIONES}

La innovación en términos FinTech puede darse lugar no solo en el área de pagos sino también en: gestión de finanzas, financiamiento, consultoría de inversiones, aplicaciones, banca digital, entre otros; su objetivo principal será el apoyo a los procesos relacionados con la banca tradicional, enfocados en soluciones principalmente para el segmento PyMEs.

El reto del gobierno ecuatoriano está en normar los procesos implicados en el ecosistema FinTech buscándose la protección del usuario, al igual que en casos de países latinoamericanos como México donde ya existe una Ley FinTech, o el de Colombia en donde existe una Asociación FinTech Nacional.

Las empresas FinTech especializadas en sistemas de pago, se sitúan en Ecuador como una alternativa accesible para las PyMEs de diversas áreas, especialmente para las dedicadas a la comercialización de productos ya que contribuye al proceso de venta. 
Las soluciones FTP, sin duda disminuyen los altos costos en los que debería incurrir una empresa que desee desarrollar una plataforma de pago por si sola.

La adopción de una alternativa FTP dependerá de la naturaleza de la empresa, y de sus directivos, quienes en base a criterios propios desarrollarán la Fase de selección de proveedor para el canal de pagos digital.

\section{REFERENCIAS CONSULTADAS}

1. Aiteco Consultores S.L. (2019). Matriz de priorización. Recuperado de Aiteco Consultores, desarrollo y gestión website: https://www.aiteco.com/matriz-depriorizacion/

2. Asociación Española para la Calidad (AEC). (2019). AEC - Matriz de priorizacion. Recuperado de AEC - Asociación Española para la Calidad website: https://www.aec.es/web/guest/centro-conocimiento/matriz-de-priorizacion

3. Banco Mundial. (2018). Inclusión financiera. Recuperado de World Bank website: http://www.bancomundial.org/es/topic/financialinclusion/overview

4. BBVA Innovation Center. (2016). Revolución fintech. Recuperado de https://www.bbva.com/wp-content/uploads/2017/08/ebook-cibbva-fintech.pdf

5. Betancourt, D. (2018). Cómo hacer una matriz de priorización paso a paso. Recuperado de Ingenio Empresa website: https://ingenioempresa.com/matriz-depriorizacion/

6. B-wise. (2019). B-wise. Recuperado de: http://www.b-wise.com

7. Cámara de Comercio de Bogotá. (2019). Un breve recorrido por la historia del fintech (p. 13). Recuperado de Cámara de Comercio de Bogotá website: https://bibliotecadigital.ccb.org.co/handle/11520/22726

8. Cantú, H. (2011). Desarrollo de una cultura de calidad (4ta.). México: McGraw Hill/ Interamericana Editores, S.A. 
9. Chishti, S., \& Barberis, J. (Eds.). (2017). The FinTech Book, The Financial Technology Handbook for Investors, Entrepreneurs and Visionaries. Recuperado de https://onlinelibrary.wiley.com/doi/pdf/10.1002/9781119218906

10. Cleri, C. (2007). El libro de las PYMES. Buenos Aires: Ediciones Granica S.A.

11. El Universo. (2019). Ecuador actualizó su Código de Comercio tras 113 años. El Universo. Recuperado de: https://www.eluniverso.com/noticias/2019/03/13/nota/7232072/ecuador-actualizosu-codigo-comercio-tras-113-anos

12. Ferraro, C., Pessoa, M., Arroio, A., Zevallos, E., Ferreira, C., Graterol, A., Rondón, M., Goldstein, E. y Kulfas, M. (2011). Apoyando a las pymes: Políticas de fomento en América Latina y el Caribe. 509. Santiago de Chile: Naciones Unidas.

13. Finnovista. (2017). Fintech Radar Ecuador identifica un ecosistema Fintech emergente en Ecuador con más de 30 startups. Recuperado de https://www.finnovista.com/fintech-radar-ecuador/

14. Fred, D. (2008). Conceptos de administración estratégica (11va.). México: Pearson Educación de México S.A.

15. González, F., Sojo, A., Carrizo, C., Morales, M., y Espíndola, N. (2018). FINTECH en el mundo. La revolución digital de las finanzas ha llegado a México. Recuperado de https://www.bancomext.com/wp-content/uploads/2018/11/LibroFintech.pdf

16. Hoder, F., Wagner, M., Sguerra, J., y Bertol, G. (2016). Cómo las innovaciones digitales están impulsando el financiamiento para las MIPYME en América Latina y el Caribe (p. 46). Olyver Wyman \& Corporación Interamericana de Inversiones ClI.

17. Igual, D. (2018). Las fintech y la reinvención de las finanzas. OIKONOMICS Revista de los Estudios de Economía y Empresa, 22-44. Recuperado de: http://oikonomics.uoc.edu/divulgacio/oikonomics/_recursos/documents/10/3_lgual _Oikonomics_10_a4_cast.pdf

18. INEC. (2017). Encuesta Nacional de Empleo, Desempleo y Subempleo-ENEMDU - TIC - 2017. Recuperado de Instituto Nacional de Estadística y Censos website: http://www.ecuadorencifras.gob.ec/documentos/webinec/Estadisticas_Sociales/TIC/2017/Tics\%202017_270718.pdf 
19. Inter-American Development Bank (IDB) \& Finnovista. (2017). FINTECH: Innovations You May Not Know were from Latin America and the Caribbean. Recuperado de: http://dx.doi.org/10.18235/0000703

20. Kushki. (2019). Kushki, La solución para tus pagos. Recuperado de Kushki website: https://www.kushkipagos.com/

21. Lee, I., \& Shin, Y. J. (2018). Fintech: Ecosystem, business models, investment decisions, and challenges. Business Horizons, 61(1), 35-46. Recuperado de: https://doi.org/10.1016/j.bushor.2017.09.003

22. Lee, K., y Larry, R. (2000). Administración de operaciones: estrategia y análisis (5ta. edición). México: Pearson Educación.

23. López, J., Langthaler, J., Fabian, M., y Mayorga, J. (2017). Una perspectiva general de Fintechs, sus beneficios y sus riesgos (p. 45). Recuperado de Asociación de Supervisores Bancarios de las Américas - ASBA website: http://www.asbasupervision.com/es/bibl/i-publicaciones-asba/i-2-otrosreportes/1602-orep24/file

24. Maldonado, F., \& Naranjo, C. (2017). Ranking financiero (p. 52). Ecuador: Revista EKOS.

25. Martínez, L., \& El Kadi, O. (2019). Logística Integral y Calidad Total, Filosofía de Gestión Organizacional orientadas al cliente. Revista Arbitrada Interdisciplinaria Koinonía, 4(7), 202-232. Recuperado de: https://doi.org/10.35381/r.k.v4i7.201

26. Maya, K. (2019). Innovación para el desarrollo económico del Gremio Artesanal. Revista Arbitrada Interdisciplinaria Koinonía, 4(7), 88-102. Recuperado de: https://doi.org/10.35381/r.k.v4i7.195

27. McWaters, R., \& Bruno, G. (2017). Beyond Fintech: A Pragmatic Assessment of Disruptive Potential in Financial Services [Part of the future of financial services series / Prepared in collaboration with Deloitte.]. Recuperado de World Economic Forum website: http://www3.weforum.org/docs/Beyond_Fintech_A_Pragmatic_Assessment_of_Disruptive_Potential_in_Financial_Services.pdf

28. Orueta, I., Echagüe, M., Bazerque, P., Correa, A., García, C., García, D., ... Rodríguez, S. (2017). La financiación de las micro, pequeñas y medianas empresas a través de los mercados de capitales en lberoamérica (4ta.; Fundación Instituto Iberoamericano de Mercados de Valores (IIMV), Ed.). Recuperado de https://www.iimv.org/iimv-wp-10/resources/uploads/2017/03/estudiocompleto.pdf 
29. Payphone. (2019). PayPhone: Cobra y paga a través de tu celular. Únete a la red del futuro. Recuperado de Payphone website: https://ivepayphone.com/

30. Rodríguez, R. (2019). Veto al Código de Comercio. Expreso.ec. Recuperado de: https://www.expreso.ec/economia/veto-codigo-comercio-psc-suma-HN2690867

31. Tundidor, A. (2017). Cómo innovar en las PYMES, Manual de mejora a través de la innovación (2da.). México: Alfaomega Grupo Editor, S.A.

32. Urueta, L. (2019). Estrategias de enseñanza y el uso de las tecnologías de información y comunicación en las instituciones educativas departamentales en el Municipio Zona Bananera - Colombia. Revista Arbitrada Interdisciplinaria Koinonía, 4(7), 185-201. Recuperado de: https://doi.org/10.35381/r.k.v4i7.200

33. Villagra, J. (2016). Indicadores de gestión, Un enfoque práctico. México: Cengage Learning Editores,S.A.

34. Villaseca, D. (2016). Digitaliza tu negocio. Madrid: ESIC Editorial.

\section{REFERENCES CONSULTED}

1. Aiteco Consultores S.L. (2019). Priorization matrix. Recovered from Aiteco Consultores, development and management website: https://www.aiteco.com/matriz-de-priorizacion/

2. Spanish Association for Quality (AEC). (2019). AEC - Priorization matrix. Recovered from AEC - Spanish Association for Quality website: https://www.aec.es/web/guest/centro-conocimiento/matriz-de-priorizacion

3. World Bank. (2018). Financial inclusion Retrieved from World Bank website: http://www.worldbank.org/en/topic/financialinclusion/overview

4. BBVA Innovation Center. (2016). Fintech revolution. Retrieved from https://www.bbva.com/wp-content/uploads/2017/08/ebook-cibbva-fintech.pdf

5. Betancourt, D. (2018). How to make a prioritization matrix step by step. Recovered from Ingenio Empresa website: https://ingenioempresa.com/matrizde-priorizacion/

6. B-wise (2019). B-wise Retrieved from: http://www.b-wise.com 


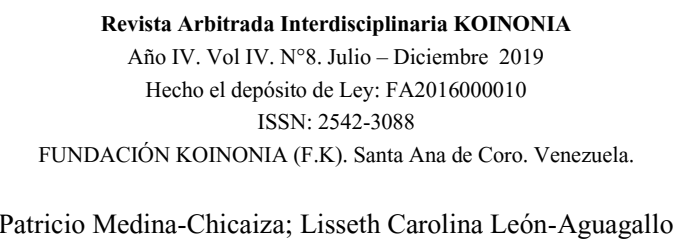

7. Chamber of Commerce of Bogotá. (2019). A brief tour of the history of fintech (page 13). Recovered from the Chamber of Commerce of Bogotá website: https://bibliotecadigital.ccb.org.co/handle/11520/22726

8. Cantú, H. (2011). Development of a quality culture (4th). Mexico: McGraw Hill / Interamericana Editores, S.A.

9. Chishti, S., \& Barberis, J. (Eds.). (2017). The FinTech Book, The Financial Technology Handbook for Investors, Entrepreneurs and Visionaries. Retrieved from https://onlinelibrary.wiley.com/doi/pdf/10.1002/9781119218906

10. Cleri, C. (2007). The book of SMEs. Buenos Aires: Ediciones Granica S.A.

11. The universe. (2019). Ecuador updated its Commercial Code after 113 years. The universe.

Recovered

from: https://www.eluniverso.com/noticias/2019/03/13/nota/7232072/ecuador-actualizosu-codigo-comercio-tras-113-anos

12. Ferraro, C., Pessoa, M., Arroio, A., Zevallos, E., Ferreira, C., Graterol, A., Rondon, M., Goldstein, E. and Kulfas, M. (2011). Supporting SMEs: Promotion policies in Latin America and the Caribbean. 509. Santiago de Chile: United Nations.

13. Finnovista (2017). Fintech Radar Ecuador identifies an emerging Fintech ecosystem in Ecuador with more than 30 startups. Recovered from https://www.finnovista.com/fintech-radar-ecuador/

14. Fred, D. (2008). Concepts of strategic administration (11th.). Mexico: Pearson Education of Mexico S.A.

15. González, F., Sojo, A., Carrizo, C., Morales, M., and Espíndola, N. (2018). FINTECH in the world. The digital revolution of finance has arrived in Mexico. Recovered from https://www.bancomext.com/wp-content/uploads/2018/11/LibroFintech.pdf

16. Hoder, F., Wagner, M., Sguerra, J., and Bertol, G. (2016). How digital innovations are driving financing for MSMEs in Latin America and the Caribbean (page 46). Olyver Wyman \& Inter-American Investment Corporation - IIC.

17. Same, D. (2018). The fintech and the reinvention of finances. OIKONOMICS Journal of Economics and Business Studies, 22-44. Retrieved from: 
http://oikonomics.uoc.edu/divulgacio/oikonomics/ recursos/documents/10/3 lgual Oikonomics 10 a4 cast.pdf

18. INEC. (2017). National Survey of Employment, Unemployment and Underemployment-ENEMDU - TIC - 2017. Retrieved from the National Institute of Statistics and Censuses website: http://www.ecuadorencifras.gob.ec/documentos/webinec/Estadisticas_Sociales/TIC/2017/Tics \% 202017_270718.pdf

19. Inter-American Development Bank (IDB) \& Finnovista. (2017). FINTECH: Innovations You May Not Know were from Latin America and the Caribbean. Retrieved from: http://dx.doi.org/10.18235/0000703

20. Kushki (2019). Kushki, The solution for your payments. Recovered from Kushki website: https://www.kushkipagos.com/

21.Lee, I., \& Shin, Y. J. (2018). Fintech: Ecosystem, business models, investment decisions, and challenges. Business Horizons, 61 (1), 35-46. Retrieved from: https://doi.org/10.1016/j.bushor.2017.09.003

22. Lee, K., and Larry, R. (2000). Operations management: strategy and analysis (5th edition). Mexico: Pearson Education.

23. López, J., Langthaler, J., Fabian, M., and Mayorga, J. (2017). An overview of Fintechs, its benefits and its risks (p.45). Recovered from the Association of Banking Supervisors of the Americas - ASBA website: http://www.asbasupervision.com/en/bibl/i-publicaciones-asba/i-2-otrosreportes/1602-orep24/file

24. Maldonado, F., \& Naranjo, C. (2017). Financial ranking (p.52). Ecuador: EKOS Magazine.

25. Martínez, L., \& El Kadi, O. (2019). Integral Logistics and Total Quality, Organizational Management Philosophy oriented to the client. Interdisciplinary Arbitrated Journal Koinonía, 4 (7), 202-232. Retrieved from: https://doi.org/10.35381/r.k.v4i7.201

26. Maya, K. (2019). Innovation for the economic development of the Artisanal Guild. Journal Interdisciplinary Arbitrated Koinonía, 4 (7), 88-102. Retrieved from: https://doi.org/10.35381/r.k.v4i7.195 
27. McWaters, R., \& Bruno, G. (2017). Beyond Fintech: A Pragmatic Assessment of Disruptive Potential in Financial Services [Part of the future of financial services series / Prepared in colla

28. Orueta, I., Echagüe, M., Bazerque, P., Correa, A., García, C., García, D., ... Rodríguez, S. (2017). The financing of micro, small and medium enterprises through capital markets in Ibero-America (4th, Fundación Instituto Iberoamericano de Mercados de Valores (IIMV), Ed.). Retrieved from https://www.iimv.org/iimv-wp-1-0/resources/uploads/2017/03/estudiocompleto.pdf

29. Payphone. (2019). PayPhone: Charge and pay through your cell phone. Join the network of the future. Retrieved from Payphone website: https://livepayphone.com/

30. Rodríguez, R. (2019). I veto the Commercial Code. Express.ec. Recovered from: https://www.expreso.ec/economia/veto-codigo-comercio-psc-suma-HN2690867

31. Tundidor, A. (2017). How to innovate in SMEs, Manual of improvement through innovation (2nd). Mexico: Alfaomega Grupo Editor, S.A.

32. Urueta, L. (2019). Teaching strategies and the use of information and communication technologies in departmental educational institutions in the Zona Bananera Municipality - Colombia. Interdisciplinary Arbitrated Journal Koinonía, 4 (7), 185-201. Retrieved from: https://doi.org/10.35381/r.k.v4i7.200

33. Villagra, J. (2016). Management indicators, A practical approach. Mexico: Cengage Learning Editores, S.A.

34. Villaseca, D. (2016). Digitize your business Madrid: ESIC Editorial. 